\title{
Comminuted fractures of the radial head treated by the Judet floating radial head prosthesis
}
A. Dotzis,
G. Cochu,
C. Mabit,
J. L. Charissoux,
J. P. Arnaud

From Department of Orthopaedic Surgery, Dupuytren Hospital University, Limoges, France

\footnotetext{
A. Dotzis, MD, Orthopaedic Surgeon

In C. Mabit, MD, Orthopaedic Surgeon, Professor J. L. Charissoux, MD, PhD, Orthopaedic Surgeon,

Professor

J. P. Arnaud, MD,

Orthopaedic Surgeon,

Professor

Department of Orthopaedic and Traumatologic Surgery

Dupuytren Hospital University,

2 Avenue Martin Luther King,

87042 Limoges Cedex, France.

- G. Cochu, MD, Orthopaedic Surgeon

Clinqué des Bains, 32 rue

Thiers, 38000 Grenoble, France.

Correspondence should be sent to Dr A. Dotzis; e-mail: nadotzis@yahoo.fr
}

(C2006 British Editorial Society of Bone and Joint Surgery doi:10.1302/0301-620X.88B6. $17415 \$ 2.00$

$J$ Bone Joint Surg [Br] 2006;88-B:760-4.

Received 14 November 2005 Accepted after revision 20 February 2006

Excision is not a suitable treatment for all comminuted fractures of the radial head. In elbows where instability can be predicted, a replacement arthroplasty of the radial head is more effective. The aim of this paper was to present the medium-term results of the Judet floating radial head prosthesis.

This operation was performed on 14 patients between 1992 and 2003, of whom 12 were reviewed at a mean follow-up of five years and three months ( 1 to 12 years). The outcome was assessed using the Mayo elbow performance score and a modified Disability of Arm Shoulder Hand (DASH) questionnaire. There were six excellent results, four good, one fair and one poor, as graded by the Mayo score. The mean DASH score was 23.9/100 (0 to 65.8) 100). The only significant complication occurred in one patient who developed a severe complex regional pain syndrome. There were no patients with secondary instability of the elbow, implant loosening, cubitus valgus, osteoporosis of the capitellum, or pain in the forearm and wrist. Our experience, combined with that of other authors using this device, has encouraged us to continue using the Judet prosthesis in comminuted fractures of the elbow where instability is a potential problem.

Fractures of the radial head account for $33 \%$ of all fractures of the elbow. ${ }^{1}$ Most are simple to treat, but comminuted fractures pose a particular problem. Conservative treatment in this type of fracture leads to poor results, and internal fixation is seldom practical. ${ }^{2}$ Excision of the radial head for an isolated comminuted fracture can give good long-term results, ${ }^{3,4}$ but when such a fracture is associated with other destabilising injuries to the elbow, it is not an appropriate solution. ${ }^{5}$ Such destabilising injuries include posterior dislocation of the elbow, with or without a fracture of the coronoid process, rupture of the medial collateral ligament and disruption of the distal radio-ulnar joint. For these injuries, a replacement arthroplasty of the radial head rather than excision will help to restore stability. ${ }^{6,7}$ The Swanson silastic prosthesis (Dow Corning Corporation, Midland, Michigan) was one of the first devices to be widely used, but the long-term results showed a high rate of fracture of the prosthesis and silastic synovitis. ${ }^{8,9}$

We chose the Judet prosthesis (Tornier SAS, Saint-Ismier, France) for a number of reasons. An all-metal implant has obvious advantages over silastic, and the argument for a modular prosthesis originates from the fact that there is no clear relationship between the size of the radial head and the diameter of the intramedullary canal of the proximal radius. A floating radial head theoretically maintains a more congruous relationship between the implant and the capitellum, than one which is fixed.

The Judet floating head prosthesis (Tornier SAS) was first produced in 1988 . The early version was made from titanium and was then replaced by cobalt-chrome in 1994 . We used the version with a long, conical stem in two sizes, large $(60 \mathrm{~mm}$ x $8 \mathrm{~mm}$ maximum diameter) and small $(55 \mathrm{~mm} \times 6.5 \mathrm{~mm}$ maximum diameter). The neck of the prosthesis, which is flanged, makes an angle of $15^{\circ}$ to the stem, in order to reproduce the normal anatomy of the proximal radius. The radial 'cup' is made of polyethylene and lies within the cobalt-chrome radial head. Once articulated, the floating head has a clearance of $35^{\circ}$ from side to side, which provides good congruence with the capitellum whatever the position of flexion and extension, or pronation and supination. The radial cups come in two sizes $(19 \mathrm{~mm}$ and $22 \mathrm{~mm}$ diameter) which can fit the different sized stems.

We used the Judet floating radial head prosthesis for replacement of comminuted fractures of the radial head in selected patients. We present the results of a small series of 14 patients. 


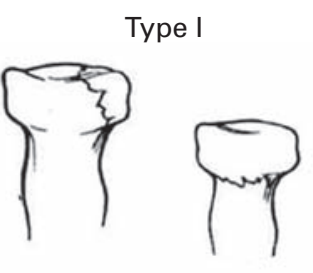

Type III
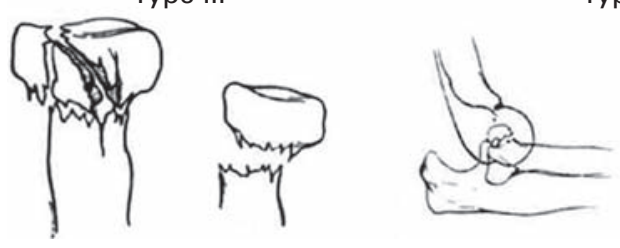

Type IV

Fig. 1

The Broberg-Morrey modification of the Mason classification. ${ }^{10}$ Type I: fracture of the radial head or neck with a displacement of $<2 \mathrm{~mm}$. Type II: fracture of the head or neck with displacement of $\geq 2 \mathrm{~mm}$. Type III: comminuted fracture of the head or neck. Type IV: dislocation of the elbow, whatever the type of the fracture.

\section{Patients and Methods}

Between October 1992 and March 2003, we used the Judet floating prosthesis in 14 patients (ten men and four women) with comminuted fractures of the radial head. Their mean age at the time of surgery was 44.8 years ( 18 to 85 ). A total of 11 patients were in active employment at the time of injury, and five of these were manual workers. The dominant limb was injured in nine patients. In every case, the mechanism of injury was a fall onto the outstretched arm, with the elbow partly flexed. The majority of the falls were domestic accidents, but one patient fell from a bicycle and one from a horse. None of the patients had sustained their fracture as a result of direct injury to the elbow.

The fractures were classified according to the BrobergMorrey modification of the Mason classification (Fig. 1). ${ }^{10}$ There were six type III and eight type IV injuries with posterior dislocation of the elbow (Fig. 2). Rupture of the medial collateral ligament was found in four elbows and disruption of the distal radio-ulnar joint in five, suggesting damage to the interosseous ligament.

The operations were performed by four different senior surgeons in our department and within eight days of injury, with the exception of two patients whose operations were performed much later, one at 120 days after injury and the other 90 days after injury. If the elbow was dislocated, the operation was preceded by manipulation under anaesthesia and reduction of the dislocation. A lateral or posterolateral approach was used. Following the excision of the fragments of the comminuted radial head, the correct size of radial head and conical stem were selected.

Following the implantation of the prosthesis, intra-operative stability of the elbow was achieved in all patients, and it was not necessary to repair the medial ligament or

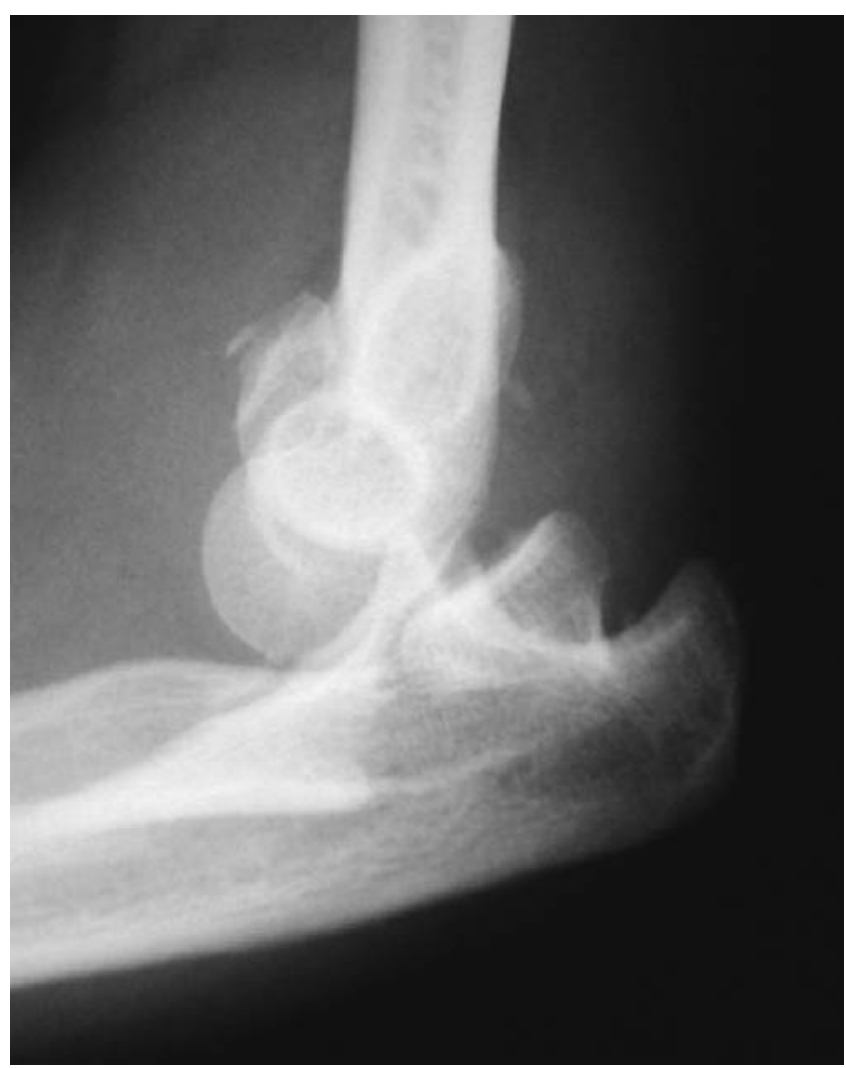

Fig. 2

Radiograph showing dislocation of the elbow with an associated fracture of the radial head.

Pain (45 points): $\quad$ None: 45

Low: 30

Moderate: 15

Severe: 0

Mobility (20 points): Range of movement $>100^{\circ}: 20$; from $50^{\circ}$ to $100^{\circ}: 15 ;<50^{\circ}: 5$

Stability (10 points): Stable: 10

Moderate: 5

Unstable: 0

Function (25 points): Hand-nape of the neck Hand-mouth

Hygiene

Dressing

To put one's shoes on

Result:

Excellent $>95$ points, good 75 to 94 points, fair 60 to 74 points, poor $<60$ points

Fig. 3

The Mayo elbow performance scoring system. ${ }^{10}$ 


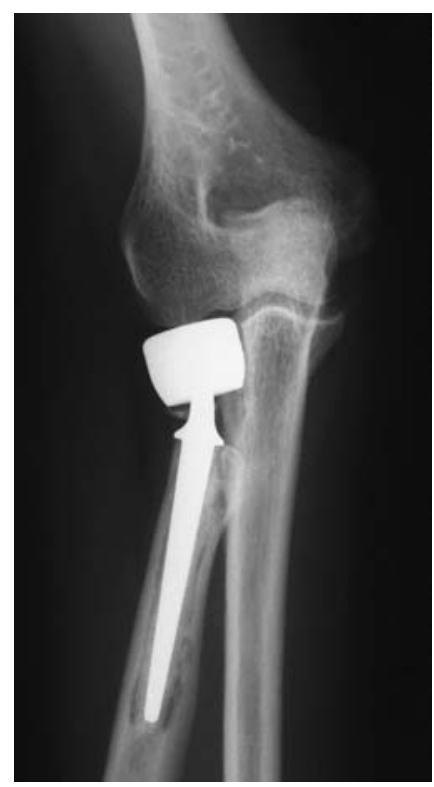

Fig. $4 a$

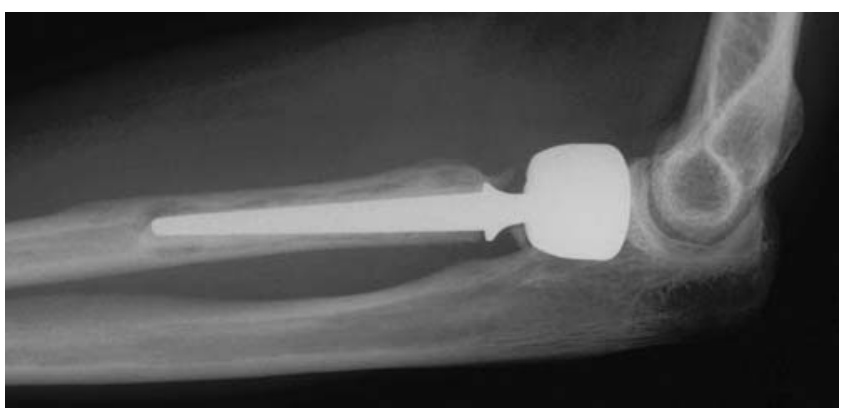

Fig. 4b

Radiolucencies appeared at a) ten months post-operatively, b) without evolution at four years.

explore on the distal radio-ulnar joint. Early mobilisation was undertaken the day after surgery. In nine patients the elbow was supported by a removable splint between physiotherapy sessions, which consisted of active and passive flexion and extension, as well as supination and pronation and continued for up to a year. The remaining patients had physiotherapy, but no splint.

All the patients were reviewed by the same surgeon (AD) and an evaluation was undertaken using the Mayo elbow performance score (Fig. 3$)^{10}$ and an adaptation of the Disability of Arm Shoulder Hand (DASH) questionaire. ${ }^{11}$ The assessment included a record of pain, stability, any cubitus valgus deformity, the range of movement at the elbow and wrist, and forearm rotation. Each range of movement was compared with the contralateral elbow. Any interference with daily living was recorded, and grip strength was measured with a dynamometer (Janar TEC, Clifton, New Jersey). Standardised anteroposterior and lateral radiographs were taken of both elbows and wrists for comparison. The radiographs were also used to look for any evidence of periarticular ossification, osteoarthritic change, osteoporosis of the capitellum, loosening of the prosthesis and disruption of the distal radio-ulnar joint.

\section{Results}

Of the 14 patients in our series, one died and one was lost to follow-up. The Judet prosthesis remained in place in the 12 remaining patients who returned for assessment at a mean of five years and three months after surgery ( 1 to 12 years). Six patients reported a pain-free elbow, five complained of pain on activity and one had permanent pain, requiring analgesia. This patient was recovering from postoperative complex regional pain syndrome.

All the elbows and wrists were stable with no cubitus valgus noted and no pain in the wrist. There was no instance of recurrent dislocation of the elbow, or dislocation of the prosthetic radial head. The range of elbow movement was excellent, with a mean arc of flexion between $14^{\circ}$ and $140^{\circ}$. Of the 12 patients, ten had full elbow flexion to $145^{\circ}$. The mean supination was $84^{\circ}\left(70^{\circ}\right.$ to $\left.90^{\circ}\right)$ and pronation $87.5^{\circ}$ $\left(70^{\circ}\right.$ to $\left.90^{\circ}\right)$. The mean grip strength was $90 \%$ that of the contralateral side $(45 \%$ to $120 \%)$. The five manual workers resumed their normal duties at a mean of 88 days ( 45 to 180) after injury, without the need for specialised rehabilitation.

The only complication occurred in the patient with complex regional pain syndrome, who complained of stiffness of the elbow and severe pain involving the whole upper limb. An arthroscopic arthrolysis of the elbow was required one year after surgery and two months later, at the time of review, she remained in pain and was still attending the clinic for rehabilitation. She had elbow flexion to $90^{\circ}$, extension of $-40^{\circ}$ and both pronation and supination of $90^{\circ}$.

There were no cases of infection. The follow-up period is too short to comment on late development of humero-ulnar osteoarthritis.

The Mayo elbow performance score ${ }^{10}$ showed that six patients had an excellent clinical result, four good, one fair and one poor result. The mean DASH ${ }^{11}$ score was 23.9/100 points (0 to 65.8/100).

The radiological review showed no evidence of elbow instability or progressive loosening of the prosthesis, though in one patient a peri-prosthetic lucency was seen at ten months after surgery. However, this had not progressed at four years follow-up (Fig. 4). Heterotopic calcification was found in seven elbows, usually on the medial side, but this finding was not of clinical significance. There were no cases of humero-ulnar arthritis, cubitus valgus or disturbance of the distal radio-ulnar index, which is consistent with the good outcome noted for the wrists.

\section{Discussion}

For comminuted fractures of the radial head where internal fixation is not possible, simple excision can give good longterm results, providing the fracture is an isolated injury and 
Table I. The principle studies demonstrating the results of the Judet floating prosthesis

\begin{tabular}{|c|c|c|c|}
\hline Authors & $\begin{array}{l}\text { Number of } \\
\text { patients }\end{array}$ & $\begin{array}{l}\text { Mean follow-up } \\
\text { (mths) }\end{array}$ & Results (Mayo classification) ${ }^{10}$ \\
\hline Judet et al ${ }^{14}$ & 12 & 49 & $\begin{array}{l}3 \text { excellent, } 2 \text { good (fresh fractures) } \\
1 \text { excellent, } 4 \text { good, } 2 \text { fair (old fractures) }\end{array}$ \\
\hline Popovic et al ${ }^{19}$ & 11 & 32 & 4 excellent, 4 good, 2 fair, 1 poor \\
\hline Smets et al ${ }^{18}$ (multicentre) & 13 & 25 & $\begin{array}{l}7 \text { excellent, } 3 \text { good, } 1 \text { fair, } 2 \text { poor } \\
\text { ( } 3 \text { painful wrist, } 1 \text { prosthesis retired) }\end{array}$ \\
\hline Holmenschlager et al ${ }^{17}$ & 16 & 19 & 2 excellent, 12 good, 1 fair, 1 poor \\
\hline This study & 12 & 63 & 6 excellent, 4 good, 1 fair, 1 poor \\
\hline
\end{tabular}

there is no other lesion affecting stability of the elbow. ${ }^{12}$ In the case of a direct injury to the outer side of the elbow, the surgeon may be confident that the damage to the radial head is isolated. In other cases uncertainty will exist. It is particularly difficult to diagnose associated damage to the interosseous membrane and radio-ulnar instability. ${ }^{13}$ Posterior dislocation of the elbow and laxity of the medial ligament are easier to diagnose, as is obvious disruption of the inferior radio-ulnar joint, which should always be part of the radiological assessment of such injuries.

The consequences of inappropriate excision of the radial head include symptomatic weakness and instability of the elbow, late cubitus valgus, secondary subluxation or dislocation of the elbow and proximal migration of the radius, which will have further consequences for the inferior radioulnar joint. In the long-term, inappropriate surgery may accelerate the onset of osteoarthritis. Therefore, replacement arthroplasty is indicated if there is definite evidence or reasonable suspicion of a co-existing destabilising injury. We consider a chondral lesion of the capitellum to be a contraindication to prosthetic replacement.

The timing of surgery may influence the outcome. ${ }^{14-16}$ Surgery was performed in our patients within eight days of injury. Late arthroplasty with no additional reconstructive procedure was performed in one patient at 90 days after injury with a good result, and in one at 120 days, with a fair outcome as judged by the Mayo score. Nevertheless, we believe that surgery is best performed within eight days of injury in order to allow early mobilisation and to avoid stiffness.

The results of our series compare with four other studies ${ }^{14,17-19}$ of a similar size in which the Mayo score has also been used (Table I). The summation of these studies suggests a degree of consistency is present when the operation is performed in different centres, with approximately $80 \%$ excellent or good results at medium-term follow-up. In particular, the prosthesis avoids the complications that might be expected if simple excision of the radial head had been performed in these patients. In our experience, use of the floating radial head prosthesis achieves stability of the elbow, and ligamentous repair is not necesssary. In our series, no patient had an associated coronoid fracture. If there is such a fracture and the elbow is not stabilised by the radial head prosthesis, we believe that internal fixation of the coronoid process is required.
Reports of monoblock metallic prostheses ${ }^{20-22}$ show medium-term functional results to be as good as with the floating prosthesis, but the radiological reviews are more worrying. The monoblocks show a high rate of radiolucency around the stem and also osteoporosis of the capitellum, indicating uneven and incongruous loading at the joint. There are floating head prostheses on the market other than the Judet. For example, the Guepar group ${ }^{23}$ have produced a similar metallic device (DePuy France SAS, Saint-Priest, France) but the cemented stem is shorter than that of the Judet prosthesis, while others have produced prostheses in which the radial head is made of different materials (KPS prosthesis; Wright Medical Technology, Arlington, Tennessee and McPyc prosthesis in polycarbon; Bioprofile SA, Grenoble, France). We found that the Judet device works well in the medium-term and only long-term comparative studies ${ }^{24}$ of current radial head prostheses will reveal if one design is superior to another.

No benefits in any form have been received or will be received from a commercial party related directly or indirectly to the subject of this article.

\section{References}

1. Mabit C. Fractures récentes et anciennes de la tête radiale. Annales Orthop de I'Ouest 1994;26:151-93.

2. King GWJ, Evans DC, Kellam JF. Open reduction and internal fixation of radial head fractures. J Orthop Trauma 1991;5:21-8.

3. Coleman DA, Blair WF, Shurr D. Resection of the radial head for fracture of the radial head: long-term follow-up of seventeen cases. J Bone Joint Surg [Am] 1987; 69-A:385-92.

4. Janssen RP, Vegter J. Resection of the radial head after Mason type III fractures of the elbow: follow-up at 16 to 30 years. J Bone Joint Surg [Br] 1998;80-B:231-3.

5. Morrey BF, Tanaka S, An KN. Valgus stability of the elbow: a definition of primary and secondary constraints. Clin Orthop 1991;265:187-95.

6. Morrey BF. Current concepts in the treatment of the fractures of the radial head, the olecranon, and the coronoid. Instr Course Lect 1995:44:175-85.

7. Sowa DT, Hotchkiss RN, Weiland AJ. Symptomatic proximal translaton of the radius following radial head resection. Clin Orthop 1995;317:106-13.

8. Harrington IJ, Tountas AA. Replacement of the radial head in the treatment of unstable elbow fractures. Injury 1981;12:405-12.

9. Worsing RA Jr, Engber WD, Lange TA. Reactive synovitis from particulate silastic. J Bone Joint Surg [Am] 1982;64-A:581-5.

10. Morrey BF. The elbow and its disorders. Second ed. St. Louis: W.B. Saunders, 1993 311-64.

11. Dubert T, Voche P, Dumontier C, Dinh A. Le questionnaire du DASH: adaptation d'un outil d'evaluation international. Chir Main 2001;20:294-302 (in French).

12. Goldberg I, Peyland J, Yosipovitch Z. Late results of excision of the radial head for an isolated fracture. J Bone Joint Surg [Am] 1986;68-A:675-9.

13. Wallace AL, Walsh WR, van Rooiken M, Hughes J, Sonnabend DH. The interosseous membrane in radio-ulnar dissociation. J Bone Joint Surg [Br] 1997;79-B:422-7.

14. Judet $T$, Garreau de Loubresse $C$, Pirious $P$, Charnley G. A floating prosthesis for radial-head fractures. J Bone Joint Surg [Br] 1996;78-B:244-9. 
15. Monin J.-0, de Cussac J.-B, Raynaud G, Letenneur J. Résultats des implants prothétiques de tête radiale. Annales Orthop de l'Ouest 1994;26:180-93.

16. Morrey BF, Askew L, Chao EY. Silastic prosthetic replacement for the radial head. $J$ Bone Joint Surg [Am] 1981;63-A:454-8.

17. Holmenschlager F, Halm JP, Winckler S. Fresh fractures with the radial head: results with the Judet prosthesis. Rev Chir Orthop Reparatrice Appar Mot 2002;88: 387-97 (in French).

18. Smets S, Govaers K, Jansen N, Van Riet R, Schaap $\mathbf{M}$. The floating radial head fractures: a multicentric study. Acta Orthop Belg 2000;66:353-8.

19. Popovic N, Gillet P, Rodriguez A, Lemaire R. Fracture of the radial head with associated elbow dislocation: results of treatment using a floating radial head prosthesis. J Orthop Trauma 2000;14:171-7.
20. Harrington IJ, Sekiy-Otu A, Barrington TW, Evans DC, Tuli V. The functional outcome with metallic radial head implants in the treatment of unstable elbow fractures: a long term review. J Trauma 2001;50:46-52.

21. Knight DJ, Rymaszewski LA, Amis AA, Miller JH. Primary replacement of the fractured radial head with a metal prosthesis. J Bone Joint Surg [Br]1993;75-B:572-6.

22. Moro JK, Werier J, MacDermid JC, Patterson SD, King G. Arthroplasty with a metal radial head for unreconstructible fractures of the radial head. J Bone Joint Surg [Am]2001;83-A:1201-11.

23. Alnot JY, Katz V, Hardy P, GUEPAR. Guepar radial head prosthesis for recent and old fractures: a series of 22 cases. Rev Chir Orthop Reparatrice Appar Mot2003;89:304-9 (in French).

24. Skalski K, Swieszkowski W, Pomianowski S, Kedzior K, Kowalik S. Radial head prosthesis with a mobile head. J Shoulder Elbow Surg 2004;13:78-85. 